\title{
Dusty space plasma diagnosis using temporal behavior of polar mesospheric summer echoes during active modification
}

\author{
A. Mahmoudian ${ }^{1}$, W. A. Scales ${ }^{1}$, M. J. Kosch ${ }^{2}$, A. Senior ${ }^{2}$, and M. Rietveld ${ }^{3}$ \\ ${ }^{1}$ The Bradley Department of Electrical and Computer Engineering, Virginia Tech, USA \\ ${ }^{2}$ Physics dept., Lancaster University, Lancaster LA1 4YB, UK \\ ${ }^{3}$ EISCAT Scientific Association, Ramfjordmoen, 9027 Ramfjordbotn, Norway
}

Received: 4 February 2011 - Revised: 13 November 2011 - Accepted: 19 November 2011 - Published: 29 November 2011

\begin{abstract}
The objective of this paper is to study the effect of different plasma and dust parameters on Polar Mesospheric Summer Echoes (PMSE) temporal behavior after turn-on and turn-off of radio wave heating and to use these responses to diagnose the properties of the dust layer. The threshold radar frequency and dust parameters for the enhancement or suppression of radar echoes after radio wave heating turn-on are investigated for measured mesospheric plasma parameters. The effect of parameters such as the electron temperature enhancement during heating, dust density, dust charge polarity, ion-neutral collision frequency, electron density and dust radius on the temporal evolution of electron irregularities associated with PMSE are investigated. The possible diagnostic information for various charged dust and background plasma quantities using the temporal behavior of backscattered radar power in active experiments is discussed. The computational results are used to make predictions for PMSE active modification experiments at 7.9, 56, 139, 224 and $930 \mathrm{MHz}$ corresponding to existing radar facilities. Data from a $2009 \mathrm{VHF}$ (224 MHz) experiment at EISCAT is compared with the computational model to obtain dust parameters in the PMSE.
\end{abstract}

Keywords. Space plasma physics (Active perturbation experiments)

\section{Introduction}

Polar Mesospheric Summer Echoes (PMSEs) are very strong radar echoes produced by structures in the electron density at half the radar wavelength. Those structures in electron density are formed due to the charging onto the subvisible irregularity structures in the dust density. These echoes have been observed typically in the $50 \mathrm{MHz}$ to $1.3 \mathrm{GHz}$ frequency range

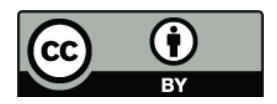

Correspondence to: A. Mahmoudian (alirezam@vt.edu) in the summer polar mesosphere (Eklund and Balsley, 1981; Rapp and Lübken, 2004; Cho and Kelley, 1993; Cho and Rottger, 1997, and references therein). The electron bite-outs observed by in-situ rocket measurements are direct evidence for the presence of charged ice aerosol particles at PMSE altitudes (Pfaff et al., 2001; Ulwick et al., 1988; Havnes et al., 1996a, b). The cause of these dust density structures and their persistence over relatively long intervals (10s to 100 s of milliseconds) has been an open scientific question (Rapp and Lübken, 2004). It has been observed in experiments that artificial perturbation of the PMSE by radio waves from a ground based ionospheric heating facility can lead to a useful diagnostic for parameters of the dust clouds (Chilson et al., 2000; Belova et al., 2001, 2003). The temporary enhancement of PMSE, described as the PMSE overshoot, was observed in experiments by Havnes et al. (2003) and Havnes (2004) and occurs when the artificial electron heating is turned off.

The first theoretical model was developed by Havnes to explain the overshoot effect after heater turn-off for VHF radar data (Havnes et al., 2003; Havnes, 2004). The original theoretical model was successfully utilized to predict this behavior and was the first major step in utilizing PMSE modification as a diagnostic tool. However, it should be noted that due to the incorporation of a Boltzmann model for electrons and ions and therefore neglecting finite diffusion time effects, the original model is not able to accurately predict the temporal evolution of modified PMSE for a range of radar frequencies and dust parameters.

Enhancement of the radar echoes after turn-off of the artificial electron heating was observed in several experiments in the UHF and VHF band (Havnes et al., 2003; Havnes, 2004; Biebricher et al., 2006; Naesheim et al., 2008). Similar behavior was observed in VHF and UHF PMSE with the same amount of reduction of backscattered power after heater turnon. The same turn-off overshoot was measured which was argued as the same generating source for both VHF and UHF PMSE. Therefore, both VHF and UHF PMSE are produced

Published by Copernicus Publications on behalf of the European Geosciences Union. 
by electron irregularity structures shaped by dust density irregularities (Naesheim et al., 2008).

$\mathrm{La} \mathrm{Hoz} \mathrm{also} \mathrm{recently} \mathrm{reported} \mathrm{the} \mathrm{first} \mathrm{joint} \mathrm{measurements}$ of PMSE, during heating using the EISCAT facility, with the EISCAT VHF radar at $224 \mathrm{MHz}$ (Bragg scale of $0.67 \mathrm{~m}$ ) and the MORRO radar at $56 \mathrm{MHz}$ (Bragg scale of $2.7 \mathrm{~m})(\mathrm{La} \mathrm{Hoz}$ et al., 2010). Weakening of the PMSE after heater turn-on was observed for both radar frequencies. Although an intensification of PMSE was measured by MORRO radar over some intervals after heater turn-off, the enhancement of radar echoes measured by the MORRO radar was modest ( $\mathrm{La} \mathrm{Hoz}$ et al., 2010).

In recent years there has been an extensive effort to measure the size and density of ice particles in the mesosphere through in-situ measurements by rockets (Robertson et al., 2009, and special issue devoted to the measurements of ice particles), and remote measurements by lidar (Baumgarten et al., 2008), radar (Li et al., 2010) and satellite (von Savigny et al., 2005; Hervig et al., 2008). In this paper ice particle measurements described in Robertson et al. (2009) are used for our model calculations. Negative particles with radii $1.2 \mathrm{~nm}$ and positive particles with radii $0.5-1 \mathrm{~nm}$, each with a number density near $2 \times 10^{9} \mathrm{~m}^{-3}$ were measured in the altitude range $86-88 \mathrm{~km}$ (Robertson et al., 2009). The signature of the larger ice particles with radii $>3 \mathrm{~nm}$ and number density $0.5 \times 10^{9} \mathrm{~m}^{-3}$ were observed at $84-88 \mathrm{~km}$. Negative particles with a number density of $4.5 \times 10^{9} \mathrm{~m}^{-3}$ and radii $1-2 \mathrm{~nm}$ were also detected from $85-87.5 \mathrm{~km}$ during the second rocket flight (Robertson et al., 2009).

The objective of this paper is to consider the temporal behavior of electron irregularity amplitudes after turn-on and turn-off of radio wave heating based on the radar facilities and frequencies which are available at EISCAT and HAARP for actual experimental predictions. The more general work of Scales and Chen (2008) and Chen and Scales (2007) were not based on the radar facilities and frequencies available at EISCAT and HAARP nor real mesospheric parameters. The variation of plasma parameters and their effect on electron irregularity amplitude is also studied in the current work. Our investigation also utilizes more realistic dust and background plasma parameters than past work. The influence of electron density variation, as well as the size and number densities of ice particles on the turn-off overshoot characteristic curve has been considered by Havnes et al. (2004) within the context of the Boltzmann electron and ion model. In this paper, the effect of these parameters on the temporal evolution of the irregularity amplitude at different radar frequencies may be investigated since finite diffusion effects are incorporated unlike in previous work. We show that for $56 \mathrm{MHz}$ the turn-off overshoot may be suppressed in comparison with $224 \mathrm{MHz}$, which is consistent with the recent experimental data (La Hoz et al., 2010). The effect of positive ice particles observed in the in-situ experiments, electron density and ionneutral collision frequency variation and realistic parameters of mesospheric altitudes are considered in this paper which were overlooked in previous works (e.g. Scales and Chen, 2008; Chen and Scales, 2005).

The co-located EISCAT radars in Tromso, Norway, operate at 224 and $930 \mathrm{MHz}$ and the new MORRO radar ( $\mathrm{La} \mathrm{Hoz}$ and Havnes, 2008) operates at $56 \mathrm{MHz}$. HAARP in Gakona, Alaska, also has a radar that operates at $139 \mathrm{MHz}$ (Ramos et al., 2009). The possibility of observing the heater-induced PMSE turn-on overshoot over a range of dust parameters for radar frequencies of $56 \mathrm{MHz}$ and $7.9 \mathrm{MHz}$ is investigated for developing theoretical predictions for future planned experiments. The computational results are compared with the results of recent active PMSE experiments at $224 \mathrm{MHz}$. Finally, a summary and conclusion is provided.

\section{Computational model}

A hybrid computational model is used to study the temporal behavior of irregularities during active modification of PMSE (Scales and Chen, 2008; Chen and Scales, 2005). In the hybrid computational model, the electrons and ions are described with fluid equations and the dust is modeled with simulation particles using the Particle-In-Cell (PIC) method. Variation of the ion density is described by the continuity equation:

$$
\frac{\partial n_{\mathrm{i}}}{\partial t}+\frac{\partial}{\partial x}\left(n_{\mathrm{i}} v_{\mathrm{i}}\right)=P_{\mathrm{i}}+L_{\mathrm{i}}+\left.\frac{\mathrm{d} n_{\mathrm{i}}}{\mathrm{dt}}\right|_{\text {charging }}
$$

Here, $L_{\mathrm{i}}$ denotes the loss due to dissociative recombination and $P_{\mathrm{i}}$ denotes the ion production due to photoionization and energetic particle precipitation. The recombination rate is modeled using $L_{\mathrm{i}}=\alpha n_{\mathrm{e}} n_{\mathrm{i}}$ and a recombination rate coefficient $\alpha=10^{-12} \mathrm{~m}^{3} \mathrm{~s}^{-1}$ for mesopause altitudes. The term $\left.\frac{\mathrm{d} n_{\mathrm{i}}}{\mathrm{dt}}\right|_{\text {charging }}$ represents the ion density reduction due to ion flux onto the dust. An electron density of $10^{9} \mathrm{~m}^{-3}$ is used for the PMSE altitude near $85 \mathrm{~km}$ which is reasonable (Friedrich and Rapp, 2009). The electron density is determined from quasi-neutrality $n_{\mathrm{e}}(x)=n_{\mathrm{i}}(x)-\rho_{\mathrm{d}}(x) / e$ where $\rho_{\mathrm{d}}(x)$ is the dust charge density and $e$ the unit charge. Neglecting inertial effects in the momentum equation, the ion velocity can be written in this form,

$$
v_{\alpha}=\frac{1}{v_{\alpha \mathrm{n}}}\left(\frac{q_{\alpha}}{m_{\alpha}} E-\frac{K T_{\alpha}}{m_{\alpha}} \frac{\partial}{\partial x}\left(\log \left(n_{\alpha}\right)\right)\right) .
$$

Here $\alpha=e$ or $i$, and $q_{\alpha}, m_{\alpha}, T_{\alpha}$ and $v_{\alpha n}$ are the species charge, mass, temperature and collision frequency, respectively, of the plasma species with neutral particles. The mesopause temperature for both ions and electrons is taken to be $T_{\mathrm{e}}=T_{\mathrm{i}}=150 \mathrm{~K}$. Proton hydrates with mass between 59 and 109 proton masses are the dominant ion compositions at the PMSE height range. $\mathrm{O}_{2}^{+}$ions are more numerous than $\mathrm{NO}^{+} . \mathrm{NO}^{+}$and $\mathrm{O}_{2}^{+}$together can be slightly more dense than the proton hydrates at $88 \mathrm{~km}$ and above (Kopp et al., 1985). It should be noted that the variation of ion mass from 50 to 100 
proton masses does not have a significant impact on the irregularity amplitude evolution during heating. The ion-neutral collision frequency is of order $10^{5} \mathrm{~s}^{-1}$ (Lie-Svenson et al., 2003). The variation of ion-neutral collision frequency is predicted to be between $3 \times 10^{4} \mathrm{~s}^{-1}$ and $3 \times 10^{5} \mathrm{~s}^{-1}$ in the altitude range $80-90 \mathrm{~km}$ (Turunen et al., 1988) and its effect on the irregularity amplitude during active modification is studied in this paper. We investigate the temporal behavior of charged dust associated with electron irregularities during electron temperature enhancements caused by radio wave heating. Therefore, the variation of electron-neutral collision frequency and recombination rate coefficient with temperature is included in the model. The electron-neutral collision frequency temperature dependence is assumed to be $v_{\mathrm{en}} \sim T_{\mathrm{e}}$ and recombination rate dependence on temperature is taken to be $\alpha \sim T_{\mathrm{e}}^{-1 / 2}$. The dust charge is modeled using a standard continuous charging model. It has been shown that a discrete charging model has reasonably close behavior with a continuous charging model for relatively small size of the dust grains at PMSE altitudes (Chen and Scales, 2007). The time varying charge for each dust particle according to the Orbital-Motion-Limited (OML) approach (Shukla and Mamun, 2002) is given by:

$\frac{d Q_{\mathrm{d}}}{d t}=I_{\mathrm{e}}+I_{\mathrm{i}}$,

where $I_{\mathrm{e}}$ and $I_{\mathrm{i}}$ are the electron and ion current on each dust particle, respectively. These currents for the negative dust particles are given by:

$I_{\mathrm{e}}=\sqrt{8 \pi} r_{\mathrm{d}}^{2} q_{\mathrm{e}} n_{\mathrm{e}} v_{\mathrm{te}} \exp \left(-q_{\mathrm{e}} \phi_{\mathrm{d}} / K T_{\mathrm{e}}\right)$,

$I_{\mathrm{i}}=\sqrt{8 \pi} r_{\mathrm{d}}^{2} q_{\mathrm{i}} n_{\mathrm{i}} v_{\mathrm{ti}}\left(1-q_{\mathrm{i}} \phi_{\mathrm{d}} / K T_{\mathrm{i}}\right)$,

For positive dust particles, the ion and electron currents are given by:

$$
\begin{aligned}
& I_{\mathrm{e}}=\sqrt{8 \pi} r_{\mathrm{d}}^{2} q_{\mathrm{e}} n_{\mathrm{e}} v_{\mathrm{te}}\left(1-q_{\mathrm{e}} \phi_{\mathrm{d}} / K T_{\mathrm{i}}\right), \\
& I_{\mathrm{i}}=\sqrt{8 \pi} r_{\mathrm{d}}^{2} q_{\mathrm{i}} n_{\mathrm{i}} v_{\mathrm{ti}} \exp \left(-q_{\mathrm{i}} \phi_{\mathrm{d}} / K T_{\mathrm{e}}\right),
\end{aligned}
$$

Here, $r_{\mathrm{d}}$ is the dust radius, $v_{\text {te }}(i)$ electron (ion) thermal velocity and $\phi_{\mathrm{d}}$ dust floating potential. The effect of a Gaussian dust radius distribution with an RMS dust radius of $10 \mathrm{~nm}$, uniform distribution and constant dust radius on the temporal evolution of the electron irregularities during radio wave heating was investigated by Chen and Scales (2005). They showed that there is only a minor difference in the temporal behavior for these different dust distributions. The relation of the dust floating potential and the dust radius to the number of charges on the dust particle can be written in the form:

$Z_{\mathrm{d}}=\frac{4 \pi \epsilon_{0} r_{\mathrm{d}} \phi_{\mathrm{d}}}{q_{\mathrm{e}}}$

The initial uncharged dust is taken to have an irregular density of the form:

$n_{\mathrm{d}}(x)=n_{\mathrm{d} 0}\left(1+\frac{\delta n_{\mathrm{d} 0}}{n_{\mathrm{d} 0}} \sin (2 \pi m x / \ell)\right)$ where $n_{\mathrm{d} 0}$ is the undisturbed dust density, $\delta n_{\mathrm{d} 0}$ is the dust density irregularity amplitude which is set to 0.2 in the simulation, $m$ is the irregularities mode number and $l$ is the system length in this model. A zero current condition often used in ionospheric plasma studies is implemented to calculate the electrostatic field $(E)$ :

$E=\left(\frac{q_{\mathrm{e}} K T_{\mathrm{e}}}{m_{\mathrm{e}} v_{\mathrm{en}}} \frac{\partial n_{\mathrm{e}}}{\partial x}+\frac{q_{\mathrm{i}} K T_{\mathrm{i}}}{m_{\mathrm{i}} v_{\mathrm{in}}} \frac{\partial n_{\mathrm{i}}}{\partial x}-J_{\mathrm{d}}\right) /\left(\frac{q_{\mathrm{e}}^{2}}{m_{\mathrm{e}} v_{\mathrm{en}}} n_{\mathrm{e}}+\frac{q_{\mathrm{i}}^{2}}{m_{\mathrm{i}} v_{\mathrm{in}}} n_{\mathrm{i}}\right)$

where $J_{\mathrm{d}}$ is the dust current density calculated from the simulated dust particles, $v_{\mathrm{en}}$ electron-neutral collision frequency and $v_{\text {in }}$ ion-neutral collision frequency. Considering energy conservation, variation of the electron temperature in the Dregion due to interaction with the radio wave is described by:

$\frac{d T_{\mathrm{e}}}{d t}=-\frac{2}{3} e v_{\mathrm{e}} E / k_{B}-\delta v_{\mathrm{e}}\left(T_{\mathrm{e}}-T\right)$

The first term on the right-hand side denotes the electron energy increase by an external electric field and the second term is the electron energy loss from collisions with neutral particles. The effective fractional energy loss during a collision is $\delta, v_{\mathrm{e}}$ is the Gurevich's electron velocity and the effective electron-neutral collision frequency is $\nu_{\mathrm{e}}$. T denotes the effective temperature of the background species, $E$ is the electric field of the radio wave and $k_{B}$ is the Boltzmann's constant. The timescale for this heating is milliseconds and therefore in the simulation model, this is essentially considered as an instantaneous electron temperature change when the radio wave pump is turned "on" or "off".

The computational model presented here (Scales and Chen, 2008; Chen and Scales, 2007) is now solved by using different numerical algorithms. The governing fluid equations are written in the formulation described by Bernhardt et al. (1991). The continuity equation is solved using an implicit method. The new algorithms not only reduce the computational time substantially but also provide greater accuracy. The parameter regimes that have been considered in the simulation study are summarized in Table 1.

\section{Temporal behavior after pump turn-on}

Two physical processes have important effects on the initial behavior of the irregularities after turn-on of the radio wave pump: (1) charging of the electrons onto the dust and, (2) the ambipolar diffusion process. Another physical process that may have a secondary effect on the electron irregularity amplitude before heater turn-off is the recombination of electrons and ions. The recombination time $\tau_{\text {recombination }} \approx \frac{1}{\alpha n_{i 0}} \frac{1}{\sqrt{r_{\mathrm{h}}}}$ where $\alpha$ is the recombination rate and $r_{\mathrm{h}}$ is the ratio of electron temperature increase during heating. In the simulation $\alpha=10^{-12} \mathrm{~m}^{3} \mathrm{~s}^{-1}$ is assumed and 
Table 1. Range of important parameters considered in the model.

\begin{tabular}{ll}
\hline Parameter & Range \\
\hline Electron density & $10^{8}-10^{10} \mathrm{~m}^{-3}$ \\
Negative dust radius & $1-20 \mathrm{~nm}$ \\
Negative dust number density & $1-4.5 \times 10^{9} \mathrm{~m}^{-3}$ \\
Positive dust radius & $0.5-1 \mathrm{~nm}$ \\
Positive dust number density & $1-2 \times 10^{9} \mathrm{~m}^{-3}$ \\
Ion-neutral collision frequency & $10^{4}-3 \times 10^{5} \mathrm{~Hz}$ \\
The ratio of electron to ion tempera- & $2-4$ \\
ture increase & $10^{-12} \mathrm{~m}^{3} \mathrm{~s}^{-1}$ \\
Recombination rate & $3.6 \times 10^{7} \mathrm{~m}^{-3} \mathrm{~s}-1$ \\
Electron-ion production rate & Proton hydrates with \\
Ion compositions & the mass $59-109 \times \mathrm{m}_{\mathrm{p}}$ \\
\end{tabular}

the recombination time is of the order of $1000 \mathrm{~s}$. The diffusion process tends to smooth out irregularities and can be approximated by (Chen and Scales, 2005):

$\tau_{\text {diff }} \approx v_{\text {in }}\left(\frac{\lambda}{2 \pi v_{\text {thi }}}\right)^{2} \frac{1}{\left(1+\frac{T_{\mathrm{e}}}{T_{\mathrm{i}}}\left(1+\frac{z_{\mathrm{d} 0} n_{\mathrm{d} 0}}{n_{\mathrm{e} 0}}\right)\right)}$

where $v_{\text {in }}, \lambda$ and $v_{\text {thi }}$ are the ion-neutral collision frequency, irregularity wavelength and ion thermal velocity, respectively. Equation (12) shows that the diffusion timescale depends on $T_{\mathrm{e}} / T_{\mathrm{i}}$; therefore, after the radio wave heating turnon, the time decreases. For irregularities observed in the $50 \mathrm{MHz}$ range, $\tau_{\text {diff }} \sim 1 \mathrm{~s}$. The timescale for electron attachment onto the dust immediately after heater turn-on is approximated by

$$
\tau_{\mathrm{chg}} \approx \frac{1}{k n_{\mathrm{d} 0}}
$$

where $k$ can be written as $k \approx \frac{I_{\mathrm{e}}}{e n_{\mathrm{e}}}=\sqrt{8 \pi} r_{\mathrm{d}}^{2} v_{\mathrm{te} 0} \sqrt{r_{\mathrm{h}}} e^{\frac{-4.1}{r_{\mathrm{h}}}}$ using the electron charging current in Eq. (4). The constant -4.1 provides a description of the equilibrium normalized floating potential of the dust prior to the radio wave heating. As can be seen in Fig. 1, the simulation runs for $25 \mathrm{~s}$ before heater turn-on such that the plasma reaches equilibrium, which can be considered as the time interval after the previous heater "on" period when plasma relates back to its equilibrium. Prior to radio wave heating, the charging time onto uncharged dust is $\tau_{\text {chg }} \sim 1 \mathrm{~s}$ for the parameters given in Table 1. Therefore after turn-on of the radio wave heating, there is competition between the dust charging process and plasma diffusion process. Depending upon which process dominates, irregularities may be suppressed or enhanced. According to the diffusion and charging timescales, the temporal behavior of the irregularities depends on the irregularity scale size, ratio of electron temperature increase after turn-on of radio wave heating, dust radius and dust density. Therefore the turn-on overshoot is sensitive to several

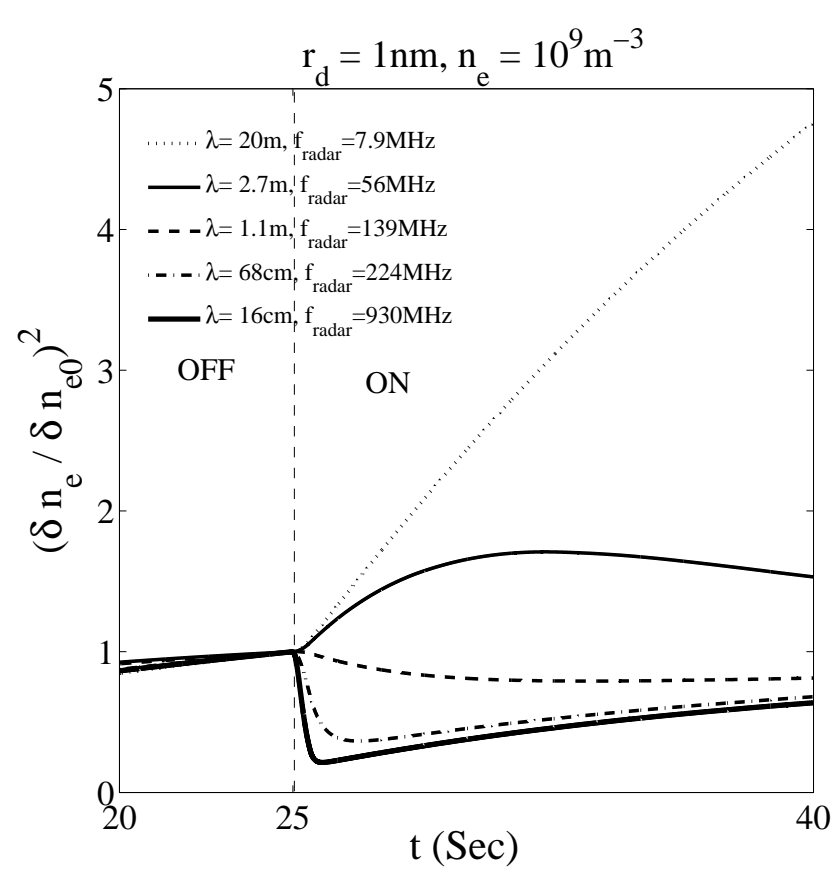

Fig. 1. The time evolution of electron irregularities during radio wave heating for varying irregularity scale size (radar frequency) utilizing the computational model. The heating is turned on at $25 \mathrm{~s}$.

parameters. The PMSE enhancement after the pump turn-on for a set of parameters may change to a suppression in the electron irregularity amplitude because of small variations of these parameters.

According to Eq. (12), the diffusion timescale is less than the charging timescale for the smaller irregularity wavelengths. After turn-on of the radio wave pump, the electron temperature starts to increase. Irregularities will diffuse out by the ambipolar diffusion process. This process suppresses the electron irregularity amplitude. For larger irregularity scale sizes, according to the Eq. (12), the diffusion time increases by a factor of $\lambda^{2}$ and the charging timescale can be less than the diffusion timescale after pump turn-on. Therefore, electron irregularity amplitudes start to grow until the point at which the diffusion timescale becomes comparable to the charging timescale. After this point, the electron irregularity amplitude will be diminished to a steady state value before turn-off of the radio wave pump where the dust charge will reach a final charge state by satisfying the equilibrium condition $I_{\mathrm{e}}+I_{\mathrm{i}}=0$.

Figure 1 shows the effect of irregularity scale size on the electron irregularity amplitude. Considering the altitude region around $85 \mathrm{~km}$ for the generation of PMSE irregularities, plasma densities are assumed to be $n_{\mathrm{e}}=n_{\mathrm{i}} \equiv n_{0}=10^{9} \mathrm{~m}^{-3}$ and the dust particles are charged up negatively and have density near $2 \times 10^{9} \mathrm{~m}^{-3}$. The dust radius is assumed to be $1 \mathrm{~nm}$. The electron temperature is assumed to increase by a factor of 4 during heating by radio waves which is a reasonable 
value according to Kassa et al. (2005). It should be noted that the ratio of electron temperature increase during heating of the ionosphere by radio waves is critically dependent on altitude, and the peak heated electron temperature normally occurs below the PMSE layer, but not always (Routledge et al., 2011). As can be seen in Fig. 1, the electron irregularity amplitude increases after the pump turn-on for irregularity scale sizes of $\lambda \approx 20 \mathrm{~m}$ and $2.7 \mathrm{~m}$, approximately corresponding to radar frequencies of 7.9 and $56 \mathrm{MHz}$, respectively. The charging timescale for these two wavelengths are less than the diffusion timescale, therefore irregularities start to grow until the diffusion timescale becomes less than the charging timescale, then diffusion will be the dominant physical process and the electron irregularity amplitude starts to decrease. The irregularities peak approximately when $\tau_{\text {diff }} / \tau_{\text {chg }}$ becomes less than 1 . It should be noted that the predicted turn-on overshoot at $7.9 \mathrm{MHz}$ is very strong. For the chosen mesospheric parameters, $56 \mathrm{MHz}$ is near the threshold frequency to observe the turn-on overshoot. Using a radar frequency greater than $56 \mathrm{MHz}$, PMSE suppression after pump turn-on can be observed due to the dominant diffusion process. For shorter wavelengths of $\lambda=1.1 \mathrm{~m}, 68 \mathrm{~cm}$, and $16 \mathrm{~cm}$, corresponding to radar frequencies of approximately 139,224 and $930 \mathrm{MHz}$, respectively, the diffusion timescale is expected to reduce by a factor of $\lambda^{2}$ according to Eq. (12). In this case, the diffusion timescale initially is less than the charging timescale and the electron irregularity amplitude starts to reduce, i.e. suppression in electron irregularity can be observed after turn-on. This behavior has been observed in PMSE pumping experiments at VHF such as Belova et al. (2001, 2003). As can be seen in Fig. 1, the amount of suppression of the irregularity amplitude after heater turnon is approximately the same for 224 and $930 \mathrm{MHz}$. There is good agreement when comparing the computational results with the UHF( $930 \mathrm{MHz})$ and $\operatorname{VHF}(224 \mathrm{MHz})$ PMSE of Fig. 12 in Naesheim et al. (2008) observed during an active experiment at EISCAT. Considering the EISCAT radar facilities for $224 \mathrm{MHz}$ and $930 \mathrm{MHz}$, MORRO radar operating at $56 \mathrm{MHz}$, and $139 \mathrm{MHz}$ radar at HAARP, these results may be applicable to estimate the PMSE parameters.

The irregularity amplitude right before the pump turn-off is related to the new charge state of the dust. This new charge state depends on different parameter values, such as dust density, electron temperature increase, dust density irregularity amplitude, dust radius and plasma irregularity scale size. Measuring some of these parameters during an active experiment can be useful to find the new charge state for the dust after artificial perturbation.

Figure 2 shows the effect of electron density and ionneutral collision frequency on the irregularity amplitude. The results are for an irregularity scale size of $\lambda=2.7 \mathrm{~m}$ which roughly corresponds to a radar frequency of $56 \mathrm{MHz}$. The dust radius is assumed to be $2 \mathrm{~nm}$ and dust density $2 \times 10^{9} \mathrm{~m}^{-3}$ (Robertson et al., 2009). Typical values of electron density at mesospheric altitudes are $10^{8}-10^{9} \mathrm{~m}^{-3}$

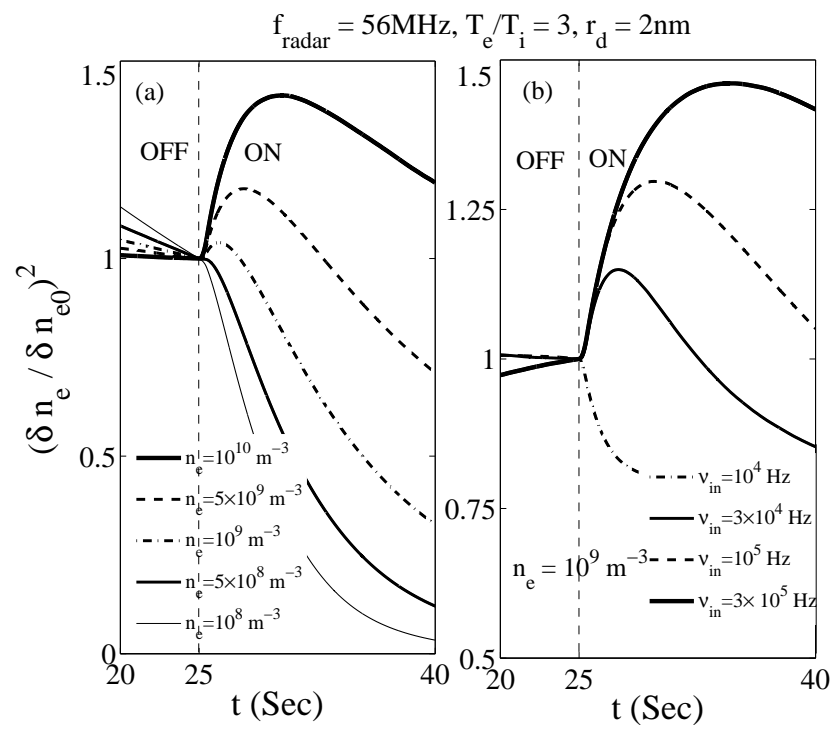

Fig. 2. The time evolution of electron irregularities during radio wave heating with varying (a) electron density and (b) ion-neutral collision frequency utilizing the computational model for a radar frequency of $56 \mathrm{MHz}$. The heating is turned on at $25 \mathrm{~s}$.

(Friedrich and Rapp, 2009) but electron density as high as $6 \times 10^{9} \mathrm{~m}^{-3}$ have also been observed (Blix, 1999). The effect of electron density variation from $10^{8}-10^{10} \mathrm{~m}^{-3}$ on the turn-on overshoot at $56 \mathrm{MHz}$ is shown in Fig. 2a. There is a turn-on overshoot for electron densities of $5 \times 10^{9} \mathrm{~m}^{-3}$ $10^{10} \mathrm{~m}^{-3}$ that may be high for this region and seen in extreme cases such as solar proton events and hard electron precipitation. For an electron density larger than $5 \times 10^{9} \mathrm{~m}^{-3}$, the charging time is less than the diffusion time after pump turnon, therefore the irregularity amplitude starts to increase until the diffusion timescale reaches a value less than the charging timescale. At this point, the irregularity amplitude will decrease due to the diffusion process which appears as a peak in the electron irregularity curve. Due to the low diffusion timescale in comparison with the dust charging timescale after the turn-on for $n_{\mathrm{e}}=10^{8}, 5 \times 10^{8} \mathrm{~m}^{-3}$ and $10^{9} \mathrm{~m}^{-3}$, the electron irregularity amplitude reduces after the pump turnon before the charging process can act to increase the electron density gradients. Therefore, electron density has a significant effect on the time at which irregularity amplitude peaks after pump turn-on.

Another parameter that can affect the temporal evolution of electron irregularity amplitude after pump turn-on is the ion-neutral collision frequency. According to Eq. (12), the diffusion timescale reduces as $v_{\text {in }}$ decreases and this may cause the diffusion process to dominate and therefore cause suppression of the irregularity amplitude after heater turnon. It has been shown that the ion-neutral collision frequency varies by an order of magnitude between $80 \mathrm{~km}\left(3 \times 10^{4} \mathrm{~Hz}\right)$ and $90 \mathrm{~km}\left(3 \times 10^{5} \mathrm{~Hz}\right)$ (Turunen et al., 1988). As can be 


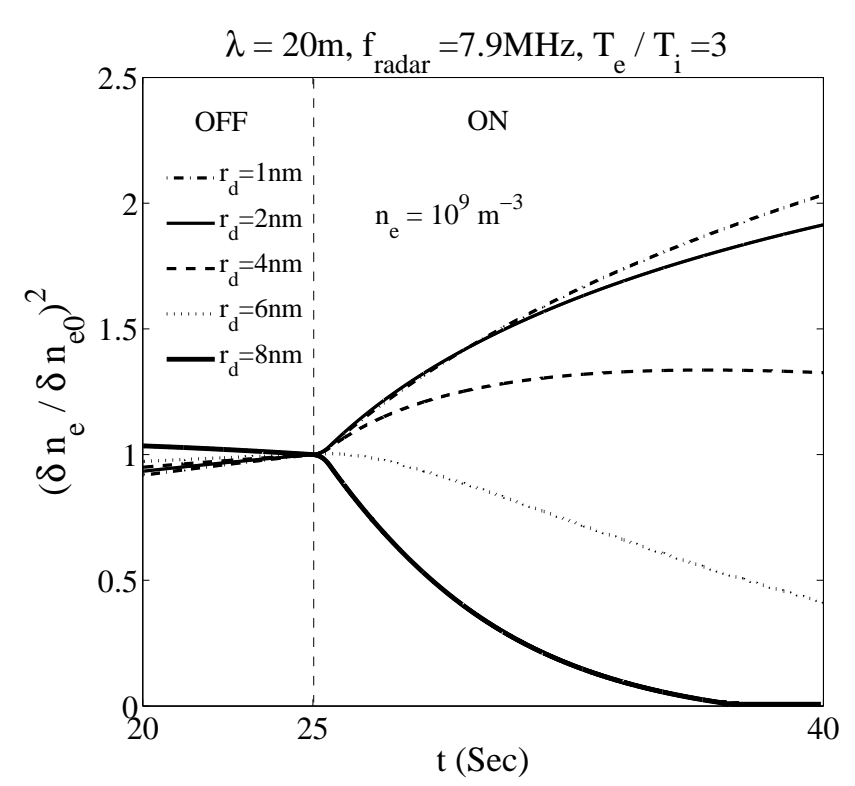

Fig. 3. The time evolution of electron irregularities during radio wave heating with varying dust radius. The radio wave pump is turned on at $25 \mathrm{~s}$.

seen in Fig. 2b, by reducing collision frequency to $10^{4} \mathrm{~Hz}$ no turn-on overshoot is expected due to the dominant diffusion process. In a recent experiment by La Hoz et al. (2010), a weakening of PMSE was observed with the EISCAT VHF radar at $224 \mathrm{MHz}$ and the MORRO radar at $56 \mathrm{MHz}$ during active modification. Comparing the results presented in Fig. 2 with this observation shows that the computational model also predicts the suppression of radar echoes for electron densities less than $10^{9} \mathrm{~m}^{-3}$ or collision frequencies less than $3 \times 10^{4} \mathrm{~Hz}$.

Another parameter which affects the evolution of irregularities after turn-on of radio wave heating is the ratio of electron temperature increase with respect to the background ion temperature. The effect of this heating ratio on the time of the irregularity peak is negligible since $\tau_{\text {diff }} / \tau_{\text {chg }}$ reaches unity approximately at the same time for all $T_{\mathrm{e}} / T_{\mathrm{i}}$ values. Another characteristic of the ratio of electron temperature increase after pump turn-on on the behavior of the irregularities is that the rate of variation of the electron irregularity amplitude in time (not shown) is larger for higher electron temperature ratios (Kassa et al., 2005). This effect can be a significant diagnostic to estimate the plasma temperate of PMSE heating by radio waves.

Figure 3 compares the effect of dust radius on the evolution of irregularities during radio wave heating in more detail. The dust density and electron density are $10^{9} \mathrm{~m}^{-3}$. According to the Bragg scattering condition, the scale size of irregularities corresponding to $7.9 \mathrm{MHz}$ is about $20 \mathrm{~m}$ and this frequency band has been the subject of recent interest (Ramos et al., 2009). For a dust radius less than $5 \mathrm{~nm}$, the turn-on overshoot can be observed. However, by increasing the dust

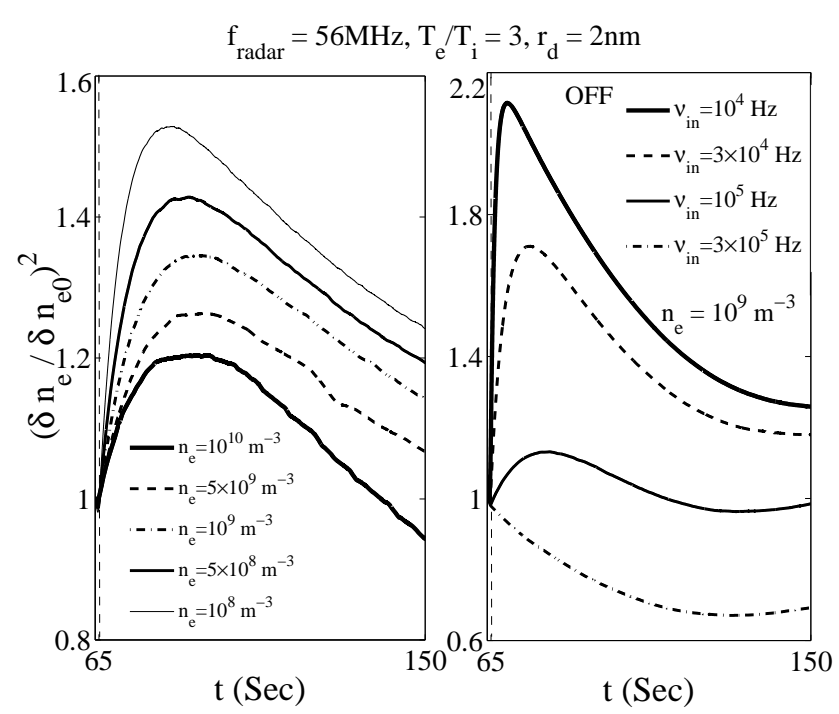

Fig. 4. The time evolution of electron irregularities after turn-off of the radio wave heating with varying (a) electron density and (b) ionneutral collision frequency utilizing the computational model for a radar frequency of $56 \mathrm{MHz}$. The heating is turned off at $65 \mathrm{~s}$.

radius to $6 \mathrm{~nm}$, the enhancement of the irregularity amplitude is substituted by a suppression.

\section{Temporal behavior after pump turn-off}

The physics after turn-off of the radio wave pump is different than the physical process after turn-on. After the radio wave is turned-off, the electron temperature returns back to its initial value before the turn-on and the ion irregularities start to diffuse out. The ion current onto the dust will be dominant and ions drag along the electrons while diffusing back. Therefore the total current onto the dust can be approximated by $\tau_{\text {chg }} \approx \frac{e}{I_{\mathrm{i}}}=\frac{1}{\sqrt{8 \pi} n_{\mathrm{i}} v_{\mathrm{i}} r_{\mathrm{d}}^{2}\left(1-q_{\mathrm{i}} \phi_{\mathrm{d}} / K T_{\mathrm{i}}\right)}$. The ion density gradient is steeper and has larger amplitude at smaller irregularity scale sizes. Therefore, ions drag along more electrons during the diffusion process and the turn-off overshoot is larger for higher radar frequencies. The computational model predicts an enhancement in electron irregularity amplitude for $\tau_{\text {diff }} / \tau_{\text {chg }}<1$ which means the electron-ion ambipolar diffusion time is less than dust particle charging time.

Figure 4 shows the temporal evolution of electron irregularities for varying electron density and ion-neutral collision frequency after the heater turn-off for PMSE at $56 \mathrm{MHz}$. As can be seen in Fig. 4a, decreasing the electron density in $50 \%$ steps from $10^{10} \mathrm{~m}^{-3}$ to $10^{8} \mathrm{~m}^{-3}$ increases the turn-off overshoot in $\sim 8 \%$ steps. According to Fig. $4 \mathrm{~b}$, increasing the ion-neutral collision frequency suppresses the turn-off overshoot and for $\nu_{\text {in }}=3 \times 10^{5} \mathrm{~Hz}$ no turn-off overshoot is observed. These results are consistent with experimental observations reported by La Hoz et al. (2010) in which a modest 


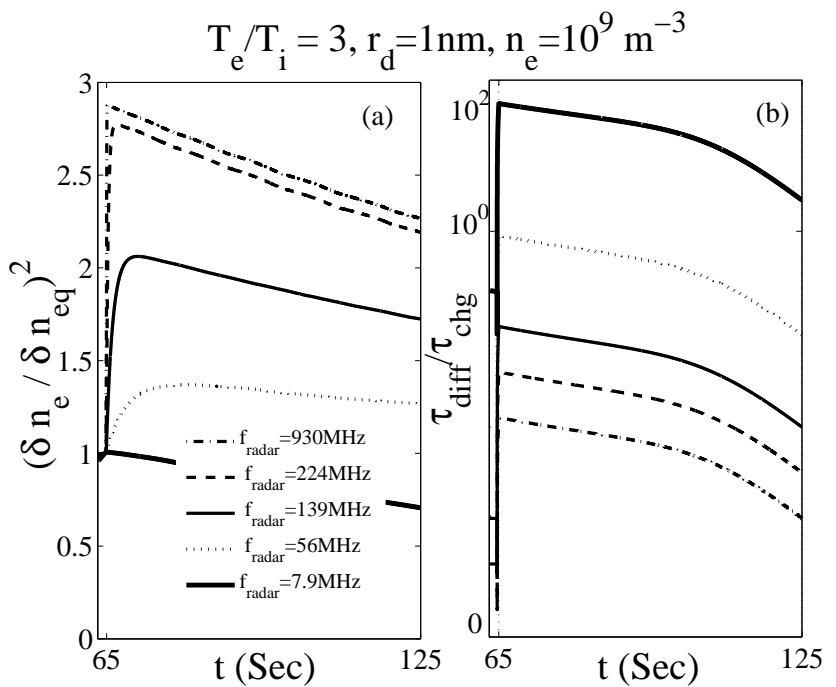

Fig. 5. (a) The time evolution of electron irregularities after turnoff of the radio wave heating with varying irregularity scale size utilizing the computational model. The heating is turned off at $65 \mathrm{~s}$. (b) Ratio of diffusion timescale to charging timescale.

turn-off overshoot at $56 \mathrm{MHz}$ was observed. The turn-off overshoot amplitude predicted by the computational model for $56 \mathrm{MHz}$ is near $20 \%$ which illustrates a weak enhancement in comparison with the turn-off overshoot observed at $224 \mathrm{MHz}$ and $930 \mathrm{MHz}$. These were reported by Naesheim et al. (2008) to be of the order of $200 \%$.

Figure 5 shows the temporal evolution of the electron irregularities after radio wave heating turn-off. As previously discussed, the turn-off overshoot can be seen for smaller irregularity scale sizes with the ratio of diffusion to charging time being approximately 1 . The turn-off overshoot was observed for UHF $(930 \mathrm{MHz})$ and VHF $(224 \mathrm{MHz})$ by Naesheim et al. (2008) in an active experiment using the EISCAT facilities. The overshoot amplitude is approximately the same for both frequencies according to Fig. 12 of Naesheim et al. (2008), which is consistent with the computational model shown in Fig. 5 for 224 and 930 MHz. The predicted turn-off overshoot amplitude is comparable with the value measured in the experiment for 224 and $930 \mathrm{MHz}$ (Naesheim et al., 2008). Figure $5 \mathrm{~b}$ shows that for smaller wavelengths, the diffusion to charging timescale ratio is much less than unity and has approximately the same amplitude at 224 and $930 \mathrm{MHz}$. Therefore the turn-off overshoot is approximately the same for these two cases. When the charging timescale becomes much smaller than the diffusion timescale, the turnoff overshoot disappears. According to Fig. 5b, for a radar frequency of $56 \mathrm{MHz}$, the charging timescale is comparable to the diffusion timescale and the turn-off overshoot amplitude reduces dramatically in comparison to the UHF and VHF PMSE turn-off overshoot for the same background parameters. In fact, $56 \mathrm{MHz}$ is near the threshold of turn-

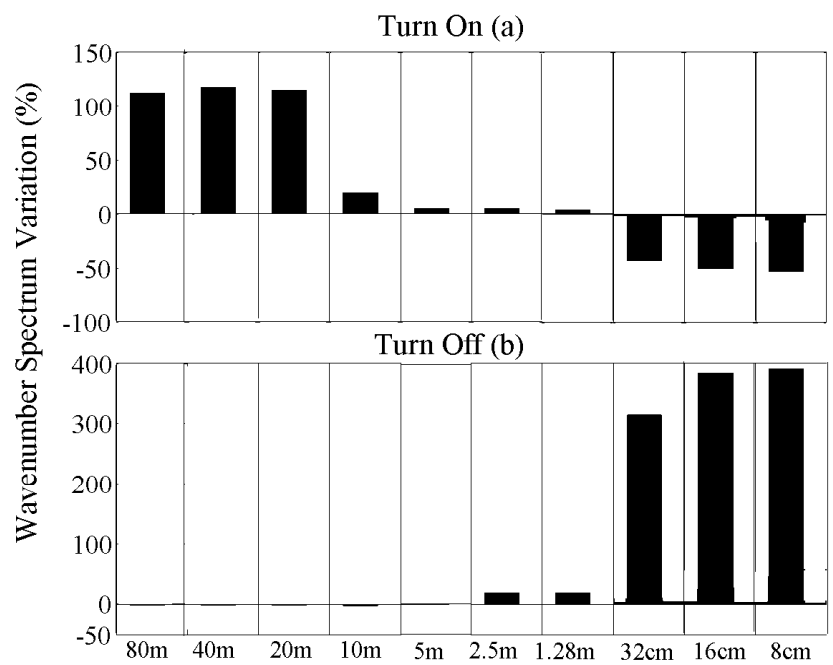

Fig. 6. The wave number spectrum for electron density fluctuation evolution, during and after radio wave heating.

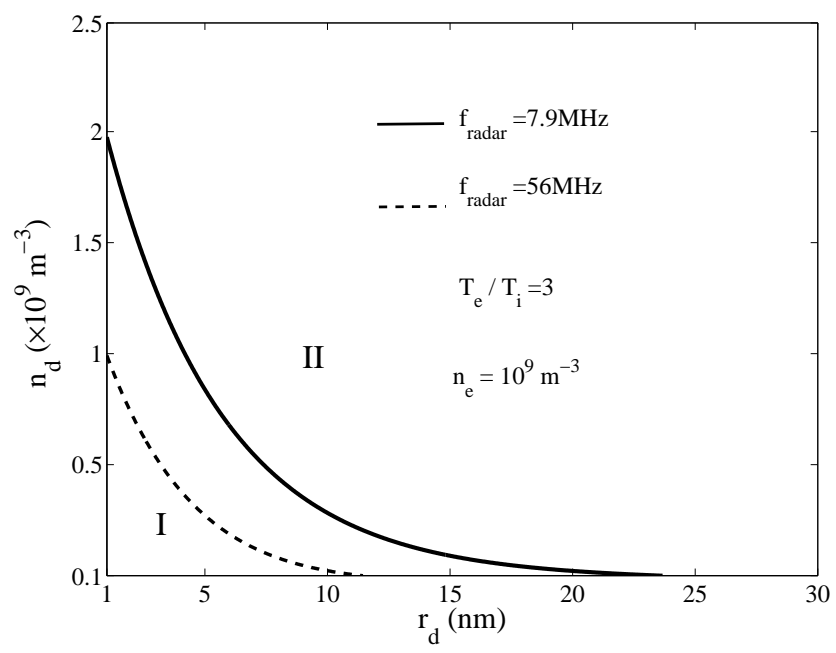

Fig. 7. The possibility of observing the turn-on overshoot. Region I shows the possible dust cloud parameter range for which turn-on overshoot is predicted to be observed. In Region II, the classic suppression of the PMSE strength after turn-on of the radio wave heating should be observed.

off overshoot and suppression after pump turn-off since the charging process becomes dominant over the diffusion process and decreases the irregularity amplitude by increasing ion current onto the dust. There is no turn-off overshoot at $7.9 \mathrm{MHz}$ which is another difference in the transient signature of PMSE after the turn-on and turn-off of radio wave heating for VHF and HF wavelengths for these parameters. It should be noted that the behavior of the turn-off overshoot with frequency is approximately described by Eq. (17) in Scales and Chen (2008).

The modeling results discussed up to this point are for the investigation of the temporal behavior of a single irregularity 
scale size. This is a useful comparison since in an actual experiment, radars operate at a single frequency and are able to look at only one irregularity scale size. However, to get more insight and a holistic picture about the temporal evolution of plasma irregularities during turn-on and turn-off of radio wave pumping in the simulation, a case consisting of 10 irregularities scale sizes is considered. The simulation box is $\lambda / \lambda_{D}=8192$ which is about $160 \mathrm{~m}$. An electron temperature enhancement during heating of the order of 10 and electron density of $10^{9} \mathrm{~m}^{-3}$ are considered. Dust density and radius are $2 \times 10^{9} \mathrm{~m}^{-3}$ and $10 \mathrm{~nm}$, respectively. The neutral dust density is extended to include a superposition of irregularities written in the form:

$n_{\mathrm{d}}(x)=n_{\mathrm{d} 0}\left(1+\sum A_{\mathrm{i}} \sin \left(\frac{2 \pi m_{\mathrm{i}} x}{l}+2 \pi \alpha_{\mathrm{i}}\right)\right)$

where $m_{\mathrm{i}}$ represents the mode number of the irregularities. $A_{\mathrm{i}}$ is a random number in the interval [0-1] and $\alpha_{\mathrm{i}}$ is a random number between -0.5 to 0.5 for each mode number produced by a random number generator. The 10 modes included correspond to irregularity scale sizes of $\lambda=0.15$, $0.31,0.62,1.25,2.5,5,10,20,40$ and $80 \mathrm{~m}$ which cover all radar frequencies from $2 \mathrm{MHz}$ to $1 \mathrm{GHz}$ according to the Bragg scattering condition. The radio wave heating is turned on at $t=25 \mathrm{~s}$ and turned-off at $t=125 \mathrm{~s}$. Figure $6 \mathrm{a}$ and $\mathrm{b}$ shows the variation of the wavenumber spectrum amplitude during radio wave heating turn "on" and "off". Figure 6a shows the difference of the wavenumber spectrum between $t=24 \mathrm{~s}$ and $26 \mathrm{~s}$, which corresponds to right before and after radio wave heating turn-on, respectively. Longer irregularity wavelengths of 80,40 and $20 \mathrm{~m}$ (lower frequencies) have about 100 percent increase in amplitude while the amplitude of shorter irregularity wavelengths are reduced by about 50 percent. This is consistent with the theory discussed before; i.e. that a turn-on overshoot is expected to happen at lower frequencies. In Fig. $6 \mathrm{~b}$ the change in the amplitude of the wavenumber spectrum from $t=124 \mathrm{~s}$ to $126 \mathrm{~s}$ is shown. Irregularity wavelengths of 32,16 and $8 \mathrm{~cm}$ have a 400 percent increase in amplitude in comparison with their amplitude before the turn-off of radio wave heating. The amplitude of longer irregularity wavelengths is suppressed by a few percent.

Figure 7 shows the possibility of observing the turn-on overshoot for radar frequencies of 56 and $7.9 \mathrm{MHz}$ for varying dust radius and density assuming the electron temperature is elevated during the heating process to $T_{\mathrm{e}} / T_{\mathrm{i}}=3$. Dust particles are assumed to be negatively charged in this calculation. The criterion for turn-on overshoot is defined as an enhancement in amplitude of at least 10 percent in irregularity amplitude for approximately $10 \mathrm{~s}$ after turn-on of the radio wave heating which should be observable during a typical radar experiment. The curves are determined by calculating several test cases with the computational model of Sect. 2 and then using curve fits through these data points. Note that this procedure may introduce a slight error of approximately
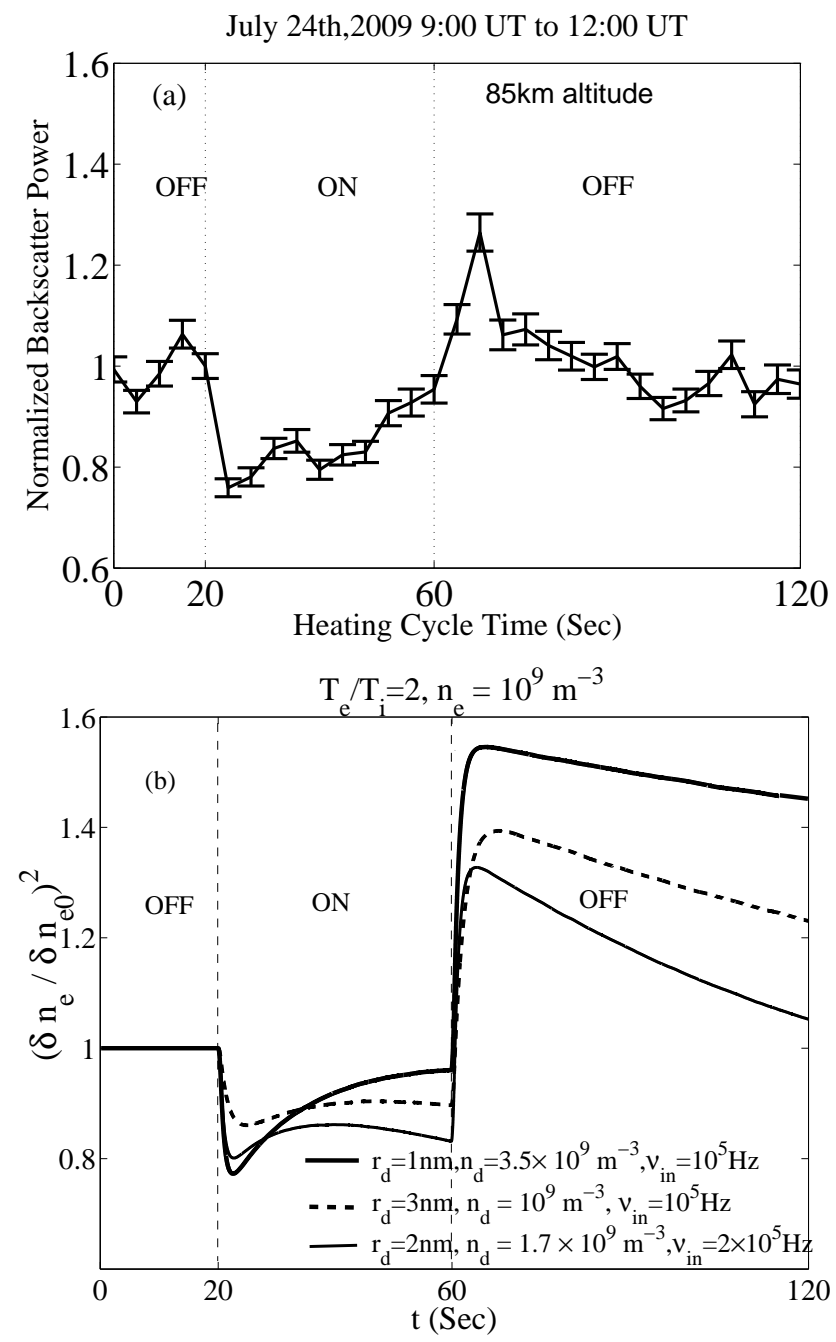

Fig. 8. A comparison of VHF radar superposed epoch data during radiowave heating and result from the computational model. Panel (a) shows the superposed epoch of PMSE VHF backscatter power, and panel (b) the computational model result.

$10 \%$ or so in the boundary curves shown. Region I in the figure, which is to the left of each curve, is the predicted parameter region for the pump turn-on overshoot to exist. In Region II, the classic suppression of the PMSE strength after turn-on of the radio wave heating should be observed. For instance, when the dust radius is $5 \mathrm{~nm}$ and radar frequency is $7.9 \mathrm{MHz}$, there is a possibility of turn-on overshoot to exist for dust densities less than $1 \times 10^{9} \mathrm{~m}^{-3}$. According to this figure, the possibility of a turn-on overshoot exists for a wider range of dust radius and density at $7.9 \mathrm{MHz}$ in comparison to $56 \mathrm{MHz}$. Note that the overshoot effect should still be observable by increasing the $T_{\mathrm{e}} / T_{\mathrm{i}}$ ratio beyond the value of 3 as shown. 


\section{Experimental data and diagnostics}

PMSE modification experiments took place at the EISCAT facility over six days from 22 to 25 and 30 to 31 July 2009 . The EISCAT facility in Tromso, northern Norway $\left(69.58^{\circ} \mathrm{N}\right.$, $19.22^{\circ} \mathrm{E}$ ), includes a $224 \mathrm{MHz}$ VHF radar. The HF transmitter at EISCAT was used for artificially heating the ionosphere.

The HF transmitter was directed vertically and operated using O-mode polarization with a frequency of $5.423 \mathrm{MHz}$ and an effective radiated power of $235 \mathrm{MW}$. A 3-min pump cycle was used for the HF radio wave heating with $40 \mathrm{~s}$ of pump on and $140 \mathrm{~s}$ of pump off. During the heating, PMSE was observed at $224 \mathrm{MHz}$ to compare the behavior of the PMSE with the computational results and get useful diagnostic information about the charged dust layer. The integrated time resolution of the VHF radar data is $4 \mathrm{~s}$ with a range resolution of $300 \mathrm{~m}$. The time step used in the model is one tenth of second.

Figure 8a shows a superposed epoch analysis of the mean averaged VHF backscatter power for 24 July from 09:00 UT to 12:00 UT which is the average of 60 heating cycles with an altitude of $85 \mathrm{~km}$. This processing is necessary to reduce the measurement errors. At $t=20 \mathrm{~s}$ the pump is switched on and subsequently the PMSE intensity instantly decreases by about 23 percent below that of the unheated level. The heating cycle is $40 \mathrm{~s}$ and at $t=60 \mathrm{~s}$ the radio wave pump is switched off. As can be seen, the PMSE intensity increases by about 30 percent above the background level which is known as turn-off overshoot (Havnes et al., 2003). The square of electron irregularity amplitude, which is related to the radar reflected power in the experiment during active modification, is shown in Fig. 8b using the computational model. The simulation is run for typical PMSE parameters. Dust density and the ratio of electron to ion temperature during radio wave heating has been adjusted to get the closest model result to the experimental data as possible. According to Robertson et al. (2009) the dust radius is assumed to be $1-3 \mathrm{~nm}$ and number density $1-4.5 \times 10^{9} \mathrm{~m}^{-3}$.

As was discussed in the first two sections, for the higher radar frequencies or smaller wavelengths like VHF and UHF, the scattering of the radar signal is expected to suppress after turn-on of the radio wave heating. Due to the short diffusion timescale, the irregularities diffuse out before the amplitude of electron irregularities can increase by electron charging of the dust under increasing electron temperature. From the discussion in the Sect. 3 about the temporal behavior of the irregularity amplitude after pump turn-on, the amount of reduction in the scattered power for the VHF radar signal is a useful means to predict the dust density.

The irregularity amplitude associated with the parameters $T_{\mathrm{e}} / T_{\mathrm{i}}=2, r_{\mathrm{d}}=1 \mathrm{~nm}, n_{\mathrm{d}}=3.5 \times 10^{9} \mathrm{~m}^{-3}, v_{\text {in }}=10^{5} \mathrm{~Hz}$ is consistent with the observed data during the pump on time interval. It should be noted that this dust density and radius are similar to that observed by in-situ experiment (Robertson et al., 2009). The computational model predicts the minimum normalized amplitude of backscattered power near 0.77 about $6.7 \mathrm{~s}$ after heater turn-on and is consistent with the experimental data that shows the minimum amplitude of normalized backscatter power about 0.78 at $6 \mathrm{~s}$ after pump turnon. The amplitude of the scattered power after heater turnoff also reflects the effect of the dust density and electron temperature enhancement during heating. The small turn-off overshoot of about 30 percent shown in Fig. 8a can be argued as a low electron temperature enhancement. In the results presented in this paper $T_{\mathrm{e}} / T_{\mathrm{i}}$ is assumed to be 2 which gives the best agreement with the data. The computational results for the parameters given above show $50 \%$ enhancement of electron irregularities after turn-off with the maximum at $t=64 \mathrm{~s}$ which is nearly the same time at which the maximum of radar echoes was observed after heater turnoff in the experiment. The irregularity amplitude associated with the parameters $T_{\mathrm{e}} / T_{\mathrm{i}}=2, r_{\mathrm{d}}=3 \mathrm{~nm}, n_{\mathrm{d}}=10^{9} \mathrm{~m}^{-3}$, and $v_{\text {in }}=10^{5} \mathrm{~Hz}$ shows a reduction in the amplitude of the turnoff overshoot and produces a faster decay which is closer to the data. In this case the dust radius has increased to $3 \mathrm{~nm}$ and density reduced which is still consistent with insitu experimental data (Robertson et al., 2009). Finally, the curve shown by the thin solid line and with parameter regime $T_{\mathrm{e}} / T_{\mathrm{i}}=2, r_{\mathrm{d}}=2 \mathrm{~nm}, n_{\mathrm{d}}=1.7 \times 10^{9} \mathrm{~m}^{-3}$, and $v_{\text {in }}=2 \times 10^{5} \mathrm{~Hz}$ shows the best agreement with the observed data during the turn-off time interval. In this case dust radius and density are assumed in between the previous two cases and the ion-neutral collision frequency is increased. According to Fig. 4 the suppression of turn-off overshoot is expected for increased collision frequency and gives better agreement with the EISCAT data. In summary, the thin solid line shows a better agreement with the observed data during the turn-off time interval. The reason that a model parameter change is needed for turn-on and turn-off to get better agreement with observed data may be because of the epoch analysis over 60 heating cycles corresponding to $3 \mathrm{~h}$ and the PMSE changing over time.

Positive dust particles have been measured at PMSE altitudes during a recent rocket experiment by Robertson et al. (2009) and such contributions of positive dust particles will most likely have an important impact on the temporal evolution of electron irregularities during turn-on and turnoff of radio wave heating. Therefore, including positively charged particles in the model may have potential to solve this inconsistency. At this time there appears to be significant uncertainty as to the charging process producing the positive dust particles and these are not consistent with the standard charging theory as described in Sect. 2. One possibility is that the positive particles grow from small molecular or cluster ions and become neutral as they grow and capture an electron, and then become negative as they capture an additional electron later (Robertson et al., 2009). A simple model is then to consider a mixture of positive, neutral, and negative dust particles in the model of Sect. 2 in which 


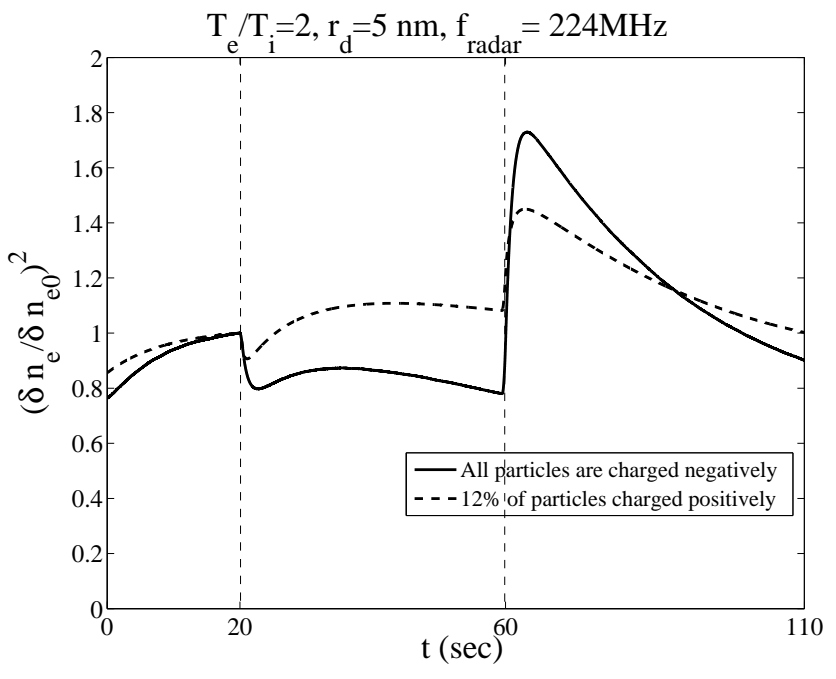

Fig. 9. The predicted effect of positively charged dust particles on the electron irregularity amplitude during PMSE heating experiments at VHF (224 MHz).

the rough approximation is used that the positive particles do not undergo dynamical charging during radio wave heating. Figure 9 shows predictions using the model of Sect. 2 for a small percentage of positive dust particles under the charging approximations just described. Figure 9 shows reduced irregularity suppression during turn-on and reduced overshoot during turn-off at VHF frequencies (e.g. 224 MHZ). There is a tendency for enhancement of the turn-on overshoot effect at HF (not shown). This behavior across the frequency range has some consistency with simply a reduction in electron density due to the addition of positive dust. These predictions are directly dependent on the charging approximation made on the positive dust which is currently unknown. As a result, incorporation of the positive dust particles may bring closer agreement between the computational and observational results of Fig. 8 particularly during turn-off, but a detailed study is beyond the scope of the current manuscript and the subject of ongoing work.

\section{Conclusions}

Modification of PMSEs by radio wave heating has significant potential for remote sensing of dust parameters in the mesosphere once suitable computational models have been developed. The work here has investigated the temporal behavior of irregularities after radio wave heating turn-on and turn-off to diagnose dusty space plasmas at mesopause altitudes. The physical process after turn-on and turn-off of radio wave heating is explained by competing diffusion and dust charging processes. Characteristics of the dusty space plasma, such as dust density and radius, charge state and plasma heating ratio, can be obtained from the temporal be- havior of the irregularities observed by radars. The VHF PMSE data from a 2009 EISCAT campaign is presented. A comparison of the experimental data and computational model has demonstrated how the absolute amplitude of the pump-induced radar backscatter overshoot features vary with important dust and plasma parameters allowing these parameters to be extracted. The possibility of observing the turn-on overshoot in the HF radar band is discussed based on typical mesospheric parameters. Most past active experiments have concentrated on VHF PMSE. It is clear from the present study that lower frequency PMSE, including HF PMSE, has substantial potential as a diagnostic tool during active modification experiments. Since the dust charging dominates during pump turn-on in the HF band it causes an enhancement of radar echoes. Further experiments should be pursued since fundamental charging physics may be revealed which is critical to understanding dusty space plasmas.

Acknowledgements. This work was supported by the National Science Foundation. The author would like to acknowledge John Janeski for helping us in proof reading of the manuscript.

Topical Editor C. Jacobi thanks two anonymous referees for their help in evaluating this paper.

\section{References}

Baumgarten, G., Fiedler, J., Lubken, F. J., and von Cossart, G.: Particle properties and water content of noctilucent clouds and their interannual variation, J. Geophys. Res., 113, 6203, doi:10.1029/2007JD008884, 2008.

Belova, E., Chilson, P. B., Rapp, M., and Kirkwood, S.: Electron temperature dependence of PMSE power, Adv. Space Res., 28, 1077-1082, 2001.

Belova, E., Chilson, P. B., Rapp, M., and Rietveld, M. T.: The response time of PMSE to ionospheric heating, J. Geophys. Res. 108, 8446, doi:10.1029/2002JD002385, 2003.

Bernhardt, P. A., Rodriguez, P., Siefring, C. L., and Lin, C. S.: Field-Aligned Dynamics of Chemically Induced Perturbations to the Ionosphere, J. Geophys. Res., 96, 13887-13900, doi:10.1029/91JA01424, 1991.

Biebricher, A., Havnes, O., Hartquist, T. W., and La Hoz, C.: On the influence of plasma absorption by dust on the PMSE overshoot effect, Adv. Space Res., 38, 2541-2550, doi:10.1016/j.asr.2005.02.061, 2006.

Blix, T. A.: Small-scale plasma and charged aerosol variations and their importance for polar mesosphere summer echoes, Adv Space Res., 24, 537-546, 1999.

Chen, C. and Scales, W. A.: Electron temperature enhancement effects on plasma irregularities associated with charged dust in the Earth's mesosphere, J. Geophys. Res., 110, A12313, doi:10.1029/2005JA011341, 2005.

Chen, C. and Scales, W. A.: Active Perturbation of Dust Associated Electron Irregularities in the Earth's Mesosphere: Discrete Charging Effects, IEEE Trans. Plamsa Sci., 35, 731-735, 2007.

Chilson, P. B., Belova, E., Rietveld, M. T., Kirkwood, S., and Hoppe, U.: First artificially induced modulation of PMSE using the EISCAT heating facility, Geophys. Res. Lett., 27, 38013804, 2000. 
Cho, J. Y. N. and Kelley, M. C.: Polar mesospheric summer radar echoes: Observations and current theories, Rev. Geophys., 31, 243-265, 1993.

Cho, J. Y. N. and Rottger, J.: An updated review of polar mesospheric summer echoes: Observation, theory, and their relationship to noctilucent clouds and subvisible aerosols, J. Geophys. Res., 102, 2001-2020, 1997.

Eklund, W. L. and Balsley, B. B.: Long-term observations of the Artic mesophere with MST radar at Poker Flat, Alaska, J. Geophys. Res., 86, 7775-7780, 1981.

Friedrich, M. and Rapp, M.: News from the Lower Ionosphere: A Review of Recent Developments, Surv. Geophys., 30, 525-559, 2009.

Havnes, O.: Polar Mesospheric Summer Echoes (PMSE) overshoot effect due to cycling of artificial electron heating, J. Geophys. Res., 109, A02309, doi:10.1029/2003JA010159,2004

Havnes, O., Naesheim, L. I., Hartquist, T., Morfill, G. E., Melands, F., Schleicher, B., Trøim, J., Blix, T., and Thrane, E.: Meter-scale variations of the charge carried by mesospheric dust, Planet. Space Sci., 44, 1191-1194, 1996a.

Havnes, O., Troim, J., Blix, T., Mortensen, W., Naesheim, L. I., Thrane, E., and Tonnesen, T.: First detection of charged dust particles in the Earths mesosphere, J. Geophys. Res., 101, 1083910847, 1996b.

Havnes, O., La Hoz, C., and Naesheim, L. L.: First Observations of the PMSE overshoot effect and its use for investigating the conditions in the summer mesosphere, Geophys. Res. Lett., 30, 2229, doi:10.1029/2003GL018429, 2003.

Havnes, O., La Hoz, C., Biebricher, A., Kassa, M., Meseret, T., Naesheim, I., and Zivkovic, T.: Investigation of the Mesospheric PMSE Conditions by Use of the New Overshoot Effect, Physica Scripta Volume T, 107, 70-78, 2004.

Hervig, M. E., Gordley, L. L., Stevens, M. H., Bailey, S. M., and Baumgarten, G.: Interpretation of SOFIE PMC measurements: Cloud identication and derivation of mass density, particle shape, and particle size, J. Atmos. Sol. Terr. Phys., 71, 316330, doi:10.1016/j.jastp.2008.07.009, 2008b.

Kassa, M., Havnes, O., and Belova, E.: The effect of electron bite-outs on artificial electron heating and the PMSE overshoot, Ann. Geophys., 23, 3633-3643, doi:10.5194/angeo-23-36332005, 2005.

Kopp, E., Eberhardt, P., Herrmann, U., and Björn, L.: Positive ion composition of the high latitude summer D-region with noctilucent clouds, J. Geophys. Res., 90, 13041-13051, 1985.

La Hoz, C. and Havnes, O.: Artificial modification of polar mesospheric winter echoes with an RF heater: Do charged dust particles play an active role, J. Geophys. Res., 113, D19205, doi:10.1029/2008JD010460, 2008.

La Hoz, C., Havnes, O., and Rietveld, M.: First joint measurements of the overshoot effect of Polar Mesospheric Summer Echoes (PMSE) at 54 and $224 \mathrm{MHz}$ excited by artificial electron heating: AGU Fall Meeting Abstracts, pp. C7+, 2010.

Li, Q., Rapp, M., Röttger, J., Latteck, R., Zecha, M., Strelnikova, I., Baumgarten, G., Hervig, M., Hall, C., and Tsutsumi, M.: Microphysical parameters of mesospheric ice clouds derived from calibrated observations of polar mesosphere summer echoes at Bragg wavelengths of $2.8 \mathrm{~m}$ and $30 \mathrm{~cm}$, J. Geophys. Res., 115, doi:10.1029/2009JD012271, 2010.
Lie-Svenson, O., Blix, T. A., and Hoppe, U. P.: Modeling the plasma response to small-scale aerosol particle perturbations in the mesopause region, J. Geophys. Res., 108, 8442, doi:10.1029/2002JD002753, 2003.

Naesheim, L. I., Havnes, O., and La Hoz, C.: A comparison of polar mesosphere summer echo at VHF $(224 \mathrm{MHz})$ and UHF $(930 \mathrm{MHz})$ and the effects of artificial electron heating, J. Geophys. Res., 113, D08205, doi:10.1029/2007JD009245, 2008.

Pfaff, R., Holzworth, R., Goldberg, R., Freudenreich, H., Voss, H., Croskey, C., Mitchell, J., Gumbel, J., Bounds, S., Singer, W., and Latteck, R.: Rocket probe observations of electric field irregularities in the polar summer mesosphere, 2001 Geophys. Res. Lett., 28, 1431-1434, doi:10.1029/2000GL012677, 2001.

Ramos, C., Kelley, M. C., Djuth, F. T., Groves, K. M., Murayama, Y., Kawamura, S., and Thorsen, D.: Multifrequency observations of Polar Mesosphere Summer Echoes using Alaskan radar facilities: Comparisons and scattering calculations, Radio Sci., 44, RS5011, doi:10.1029/2008RS004102, 2009.

Rapp, M. and Lübken, F.-J.: Polar mesosphere summer echoes (PMSE): Review of observations and current understanding, Atmos. Chem. Phys., 4, 2601-2633, doi:10.5194/acp-4-2601-2004, 2004.

Robertson, S., Horányi, M., Knappmiller, S., Sternovsky, Z., Holzworth, R., Shimogawa, M., Friedrich, M., Torkar, K., Gumbel, J., Megner, L., Baumgarten, G., Latteck, R., Rapp, M., Hoppe, U.-P., and Hervig, M. E.: Mass analysis of charged aerosol particles in NLC and PMSE during the ECOMA/MASS campaign, Ann. Geophys., 27, 1213-1232, doi:10.5194/angeo-271213-2009, 2009.

Routledge, G., Kosch, M. J., Senior, A., Kavanagh, A. J., McCrea, I. W., and Rietveld, M. T.: A statistical survey of electron temperature enhancements in heater modulated polar mesospheric summer echoes at EISCAT, J. Atmos. Sol.-Terr. Phys., 73, 472482, 2011.

Scales, W. A. and Chen, C.: On initial enhancement of mesospheric dust associated plasma irregularities subsequent to radiowave heating, Ann. Geophys., 26, 2265-2271, doi:10.5194/angeo-262265-2008, 2008.

Shukla, P. K. and Mamun, A. A.: Introduction to Dusty Plasma Physics, Institute of Physics, Bristol, 2002.

Turunen, E., Collis, P. N., and Turunen, T.: Incoherent scatter spectral measurements of the summertime high-latitude D-region with the EISCAT UHF radar, J. Atmos. Terr. Phys., 50, 289, 1988.

Ulwick, J., Baker, K., Kelley, M., Balsley, B., and Ecklund, W.: Comparison of Simultaneous MST Radar and Electron Density Probe Measurements During STATE, J. Geophys. Res., 93, 6989-7000, 1988.

von Savigny, C., Petelina, S. V., Karlsson, B., Llewellyn, E. J., Degenstein, D. A., Lloyd, N. D., and Burrows, J. P.: Vertical variation of NLC particle sizes retrieved from Odin/OSIRIS limb scattering observations, Geophys. Res. Lett., 32, L07806, doi:10.1029/2004GL021982, 2005. 